\title{
Weak Synchronization of Chaotic Coupled Map Lattices
}

\author{
F. S. de San Roman, S. Boccaletti, D. Maza, and H. Mancini \\ Departamento de Fisica y Matemática Aplicada, Universidad de Navarra, Irunlarrea s/n, 31080 Pamplona, Spain
}

(Received 24 March 1998)

\begin{abstract}
Phase synchronized states can emerge in the collective behavior of an ensemble of chaotic coupled map lattices, due to a mean field interaction. This type of interaction is responsible for synchronized chaotic global activity of the lattices, while the local activity of each map remains unsynchronized. The resulting collective dynamics is called "weak synchronization." The transition to such a state is characterized in an ensemble of one-dimensional lattices of logistic maps, in terms of the distance in phase among the different lattices. Its robustness against a small difference in the map parameters is proved. We show that this phenomenon can be associated with pattern formation. [S0031-9007(98)07439-0]
\end{abstract}

PACS numbers: 05.45. $+\mathrm{b}, 47.54 .+\mathrm{r}$

Synchronizing chaotic systems means linking their trajectories to the same values at the same times, so that they remain in step with each other. The mechanism was originally proposed by Pecora and Carroll (PC) [1], who have shown the possibility for two identical chaotic systems evolving from different initial conditions to synchronize by means of the transmission of a signal, provided that the sub-Liapunov exponents of the subsystem to be synchronized are all negative.

On the other hand, the possibility of encoding a message within a chaotic dynamics has also been shown [2]. This achievement, in connection with the original PC idea, has stimulated further investigations in order to produce secure communication between a message sender and a message receiver [3].

Most recently, the concept of chaos synchronization has been extended to that of phase synchronization of chaotic systems [4]. In this process, the interaction of nonidentical chaotic systems can lead to a perfect locking of their phases, whereas their amplitudes remain uncorrelated. The transition to phase synchronization behavior of two coupled oscillators has been characterized with reference to the Rössler system [5,6].

All of the above body of literature refers to synchronization behavior of confined systems, i.e., systems modeled by ordinary differential equations. Synchronization of chaos or, more generally, phase locking of chaotic signals in large populations of coupled dynamical units, where each separate unit may reside on a chaotic attractor, is currently a subject of active investigations [7,8].

When dealing with populations of coupled dynamical systems, sycnhronization behavior can affect either the global activity of a population or the local activity of each element of such a population. In Ref. [8], synchronization of the collective chaotic evolution of the populations is found as a direct consequence of local synchronization of each single element of one population to the corresponding elements of the other populations. Therefore, both global and local activity undergo chaos synchronization at once. This process will be called "strong synchronization."
The aim of this Letter is twofold. The first is to demonstrate that phase synchronization behavior can be found in spatially extended systems (an ensemble of chaotic coupled map lattices) due to a global coupling on each element of the lattice. The second is to characterize this new dynamical regime which shows phase synchronization of the global activity of the lattices, while the local activity of each map remains unsynchronized. Therefore, the emerging collective behavior will be called "weak synchronization" (WS).

Let us consider an ensemble of $N$ coupled onedimensional map lattices, each one formed by $L$ logistic maps. In this system, the state $x_{k}^{i}$ of the $k$ th map $(k=$ $1, \ldots, L)$ of the $i$ th lattice $(i=1, \ldots, N)$ evolves at time $n+1$ through the rule

$$
\begin{aligned}
x_{k}^{i}(n+1)= & \left(1-2 \varepsilon_{1}-2 \varepsilon_{2}\right) \mathcal{F}_{k}^{i}\left(x_{k}^{i}(n)\right) \\
& +\varepsilon_{1} \mathcal{F}_{k}^{i}\left(x_{k-1}^{i}(n)\right)+\varepsilon_{1} \mathcal{F}_{k}^{i}\left(x_{k+1}^{i}(n)\right) \\
& +\varepsilon_{2} \mathcal{F}_{k}^{i}\left(\mathcal{M}^{i-1}(n)\right)+\varepsilon_{2} \mathcal{F}_{k}^{i}\left(\mathcal{M}^{i+1}(n)\right) .
\end{aligned}
$$

In Eq. (1), $\varepsilon_{1}, \varepsilon_{2}$ are real coupling parameters, $\mathcal{F}_{k}^{i}$ is the logistic map defined by $\mathcal{F}_{k}^{i}(x)=\mu_{k}^{i} x(1-x) ; 0<\mu_{k}^{i} \leq$ 4 [9], and $\mathcal{M}^{i}(n) \equiv \frac{1}{L} \sum_{k=1}^{L} x_{k}^{i}(n)$ is the mean activity of the $i$ th lattice at time $n$.

When $\varepsilon_{1}=\varepsilon_{2}=0$, Eq. (1) describes the dynamics of $N \times L$ independent logistic maps.

As soon as $\varepsilon_{1} \neq 0$, Eq. (1) can be seen as a collection of $N$ independent one-dimensional lattices of logistic maps. The maps are now coupled within each lattice by means of a diffusive term.

Finally, $\varepsilon_{2} \neq 0$ implies a global coupling among the lattices which equally distributes on each element of one lattice the mean activity of the nearest lattices. This latter coupling will be responsible for a collective synchronization behavior of the system, giving rise to WS. WS is characterized by the fact that the global signals $\mathcal{M}^{i}(t)$ and $\mathcal{M}^{j}(t)(i \neq j)$ of two lattices undergo phase synchronization in time, but all the maps $x_{k}^{i}(t)$ and $x_{k}^{j}(t)(k=1, \ldots, L)$ 
of the lattices are uncorrelated to each other. At variance with what was previously reported in the literature [10] about mean field coupling effects within a single onedimensional map lattice, here we consider the dynamical correlations built between different one-dimensional lattices, as a result of the exchange of their mean field.

Most of the results presented below pertain to the $\varepsilon_{1}=$ 0 case. Let us then begin putting $\varepsilon_{2} \neq 0$, and suppose that all of the maps are identical, i.e., the parameters $\mu_{k}^{i}$ are all equal to the same value $\mu$. Setting $\mu>\mu_{c} \simeq 3.569946$, the maps enter the chaotic region [11]. In such a case, Eq. (1) describes the behavior of $L \times N$ identical logistic maps, all in the chaotic state, globally coupled through a mean field interaction term. In the following, we focus our attention on a system with $N=L=100$, starting from random initial conditions, and with periodic boundary conditions. We set $\mu>\mu_{c}$, and gradually increase $\varepsilon_{2}$.

For all choices of $\mu>\mu_{c}$, a sharp transition toward a WS state is observed at $\varepsilon_{2}=\varepsilon_{2, c}(\mu)$. In Fig. 1a we report the case $\mu=3.755, \varepsilon_{2}=0.019$, where the formation of two clusters of phase synchronized chaotic $\mathcal{M}$ signals is found. Phase synchronization implies that the phase distance of the signals is constant as a function of time [4,5]. Therefore, the situation reported in Fig. 1a corresponds to a fully phase synchronized state. However, in the following, we will distinguish among phase clusters, that is, clusters made by signals whose distance in phase is constantly equal to zero. In such a case, phase clusters are defined as those containing the signals which show local maxima (minima) at the same time.

The corresponding local dynamics of the maps is unsynchronized with respect to that of the corresponding maps of other lattices belonging to the same phase cluster. As an example, we report in Fig. 1b the activity of all maps at a given time within the WS regime of Fig. 1a. The pattern of local activities looks random.

In order to quantitatively characterize WS and the transition to WS, we now define the distance in phase $D_{i, j}$ between the global activity of the $i$ th and $j$ th lattices. For this purpose, let us consider the signals $\mathcal{M}^{i}(t)$ and $\mathcal{M}^{j}(t)$, and take the former signal as the reference signal for the phase. At each time $\tau_{n}$ at which $\mathcal{M}^{i}\left(\tau_{n}\right)$ displays its $n$th local minimum (maximum), we check whether $\mathcal{M}^{j}\left(\tau_{n}\right)$ is also a local minimum (maximum). If the above condition does not hold, this means that the two signals do not belong to the same phase cluster at $t=\tau_{n}$, and we add one to their phase distance by writing $D_{i, j}(n+1)=D_{i, j}(n)+1$. In the opposite case, the two signals are clustered in phase, so that their phase distance is left unchanged. We then look for the next local minimum (maximum) at time $\tau_{n+1}$. After having traveled through the signal $\mathcal{M}^{i}(t)$, we repeat the same procedure taking $\mathcal{M}^{j}(t)$ as the reference signal for the phase. The final value of $D_{i, j}$ is an integer number ranging from zero (perfect phase clustering) to $N_{i}+N_{j}$ (signals not clustered in phase), $N_{i}\left(N_{j}\right)$ being the total number of local extrema of the $i$ th ( $j$ th) lattice. (a)

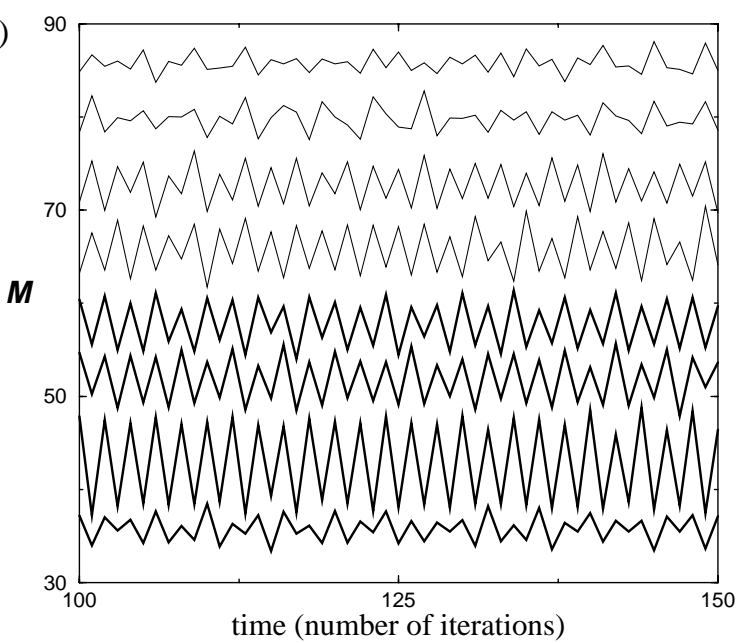

(b)

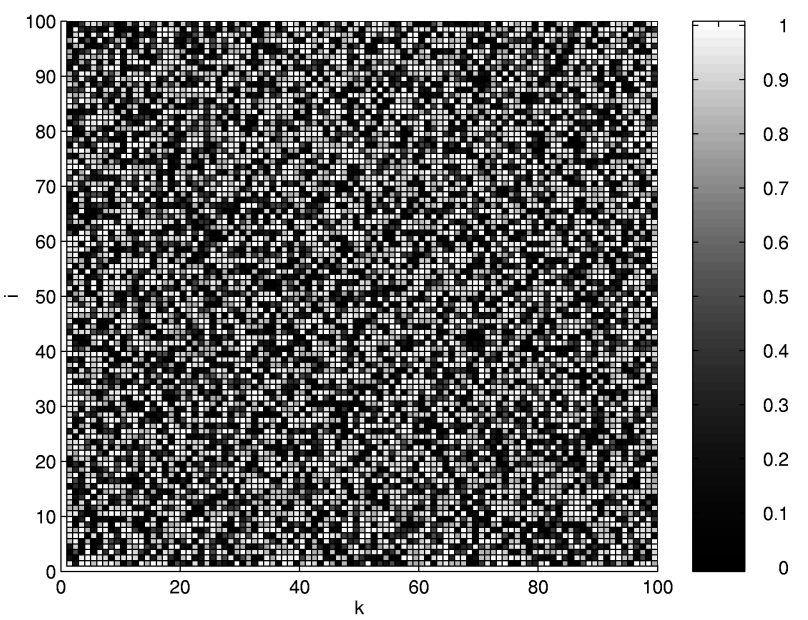

FIG. 1. (a) Temporal evolution of the global activities of the lattices. The eight displayed signals correspond to eight different lattices, forming two phase clusters (thin and thick lines). The signals have been conveniently shifted in the vertical axis, in order to highlight the phase synchronization. The scale in the $\mathcal{M}$ axis allows one to estimate the range of the chaotic fluctuations of the signals. $\mu=3.755, \varepsilon_{1}=0$, and $\varepsilon_{2}=0.019$. $N=L=100$, random initial conditions, and periodic boundary conditions. (b) Corresponding pattern of local activities of the maps, after 200 iterations within WS. The codification gray scale is reported.

The phase differences $D_{i, j}$ are distributed within a probability distribution function $\mathcal{P}(D)$, which assigns to any value of $D$ the total number of lattice couples with phase difference $D$. Such a distribution function is a good indicator of how much phase synchronization is realized in the collective behavior of our system, and of how phase synchronization is dynamically constructed.

Namely, we proceed as follows. Starting from random initial conditions, we let the system of Eq. (1) evolve for a number $N_{0}$ of iterates at $\mu=4$ (ergodic chaos [11]). Then, we increase $\varepsilon_{2}$ by a given step, and we consider as the initial condition the final state of the previous evolution of the system. We let the system evolve for other $N_{0}$ 
iterates. As soon as $\varepsilon_{2}$ becomes larger than $\varepsilon_{2, c}$, the system reaches WS. At this point, we begin decreasing the parameter $\varepsilon_{2}$ step-by-step, until again reaching phase desynchronization, always taking as initial condition of the new iteration the final state of the previous one.

At the beginning of the process, the system is not phase synchronized, and the phase differences are randomly distributed within a unimodal (single peak) function (Fig. 2a, $\left.\varepsilon_{2}=0.0172\right)$. When $\varepsilon_{2}=\varepsilon_{2, c}=0.0181$ a sharp transition is observed toward WS, which is realized by the creation of two phase clusters, each one of them formed by a large number of phase synchronized lattices. The distribution function of the phase differences is reported in Fig. $2 b$. The two peaks refer to the two clusters. Precisely, the peak at $D=0$ refers to the phase difference between two lattices of the same cluster, whereas the other peak refers to the phase difference between a lattice of one cluster and a lattice of the other. By further increasing $\varepsilon_{2}$, the two phase clusters grow, until taking all of the available lattices of the system (as can be appreciated by Fig. 2c, realized for $\varepsilon_{2}=0.0185$ ). At this point, the gradual reduction of $\varepsilon_{2}$ starts from a totally phase synchronized state.

The process of phase desynchronization appears to be very different from that of phase synchronization. From one side, the transition from WS to phase desynchronization is not sharp, but it passes through intermediate situations, where many small phase clusters appear, in addition to the main two. From the other side, global desynchronization reoccurs for $\varepsilon_{2}$ values much smaller than $\varepsilon_{2, c}$, thus revealing the presence of some hysteresis phenomenon.

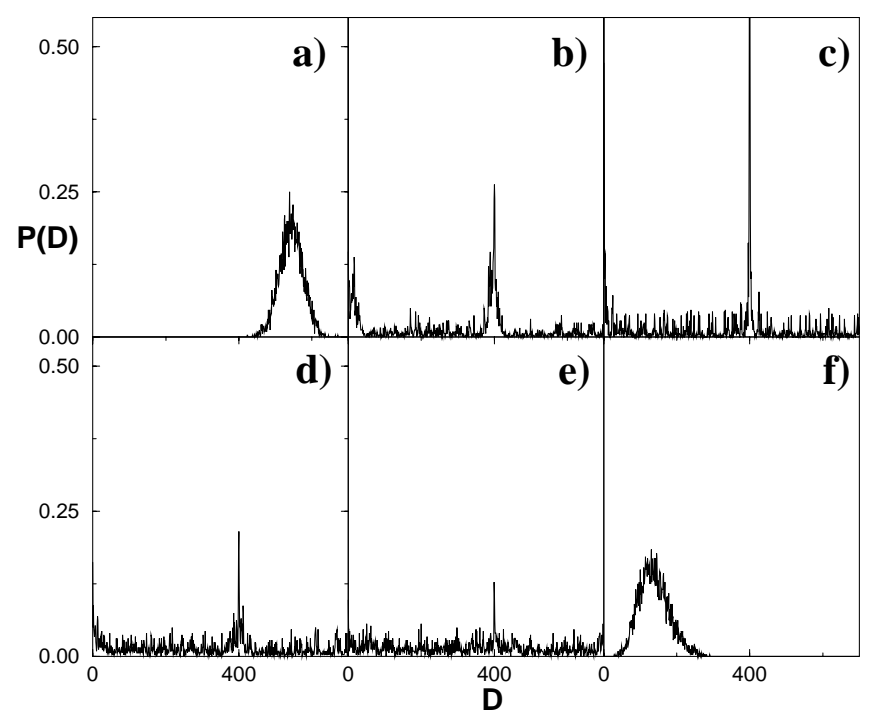

FIG. 2. Distribution functions of $D$ (see text for definition) for (a) $\varepsilon_{2}=0.0172$, (b) $\varepsilon_{2}=0.0181$, (c) $\varepsilon_{2}=0.0185$, (d) $\varepsilon_{2}=0.0182$, (e) $\varepsilon_{2}=0.0179$ (f) $\varepsilon_{2}=0.0166$. In all cases $\mu=4$. Other parameters, initial, and boundary conditions as in the caption of Fig. 1 . In all cases, the vertical axis is normalized to the value of the maximum of the histogram of (c).
The phase histograms of Figs. $2 \mathrm{~d}\left(\varepsilon_{2}=0.0182\right)$ and 2e $\left(\varepsilon_{2}=0.0179\right)$ show the formation of microclusters of phase synchronized lattices, which subtract the population to the two main clusters, and finally the transition to the phase unsynchronized state occurs at $\varepsilon_{2}=0.0166$ (Fig. 2f). Notice that the new phase distribution function is centered at a smaller $D$ value with respect to that of Fig. 2a, meaning that phase desynchronization has already occurred, but the system appears to retain some memory of the fact that it is coming from a phase synchronized state, so that the average phase difference is now smaller.

Let us introduce a new phase difference $d_{i, j}$ between lattices $i$ and $j(i \neq j)$, which is now the sum of the $L$ phase differences between each element of the $i$ th lattice and the corresponding one of the $j$ th lattice, calculated in the same way as above. The comparison between the two probability distributions for $D$ and $d$ provides a classification of the synchronization behavior. In strong synchronization [8], the two distribution functions are equal, because global synchronization is the consequence of the simultaneous local synchronization of each lattice element. However, in WS, the two distribution functions may be very different, as can be appreciated by looking at Fig. 3, which reports the distribution function of $d$ for $\mu=4, \varepsilon_{2}=0.0185$. This distribution function should be compared with that of Fig. 2c. While the latter is a signature of phase synchronized global activities, the former does not show any synchronization feature.

The scenario described above is generally observed for any $\mu>\mu_{c}$.

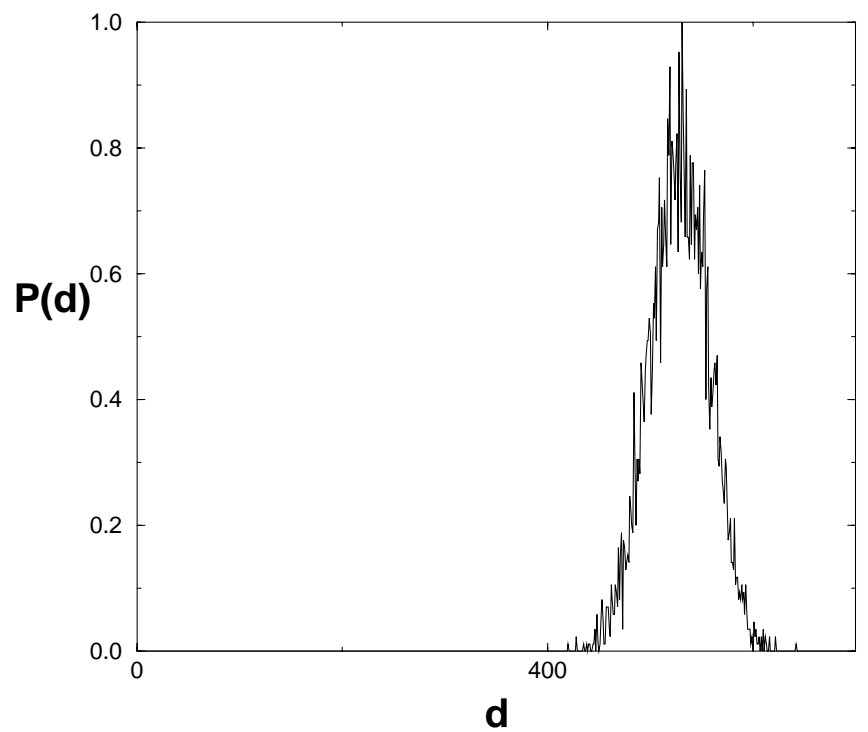

FIG. 3. Distribution function of $d$ (see text for definition) for $\mu=4, \varepsilon_{1}=0$, and $\varepsilon_{2}=0.0185$ [same situation as in Fig. 2(c)]. While the lattices are phase synchronized [Fig. 2(c)], each map of the lattices is unsynchronized. The vertical axis has been conveniently normalized to the maximum value of the histogram. 


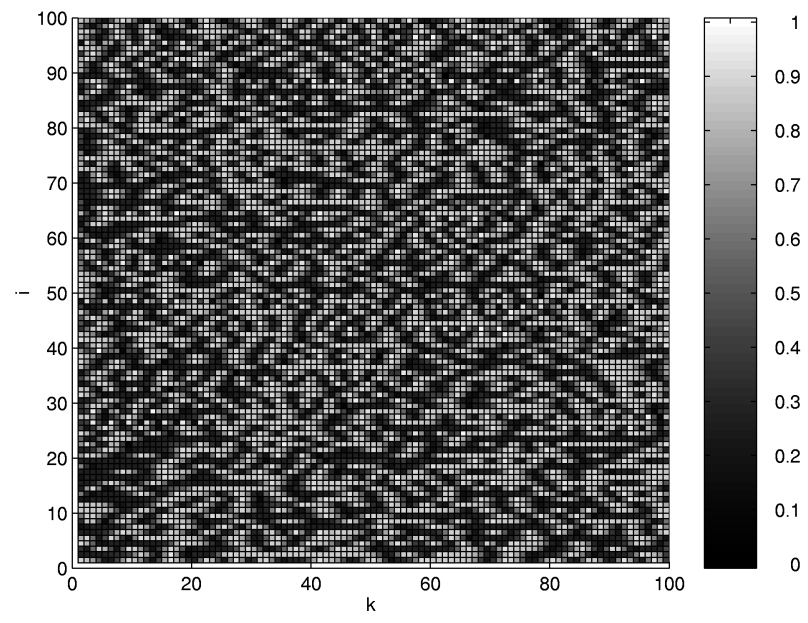

FIG. 4. WS associated with the emergence of twodimensional patterns in the local activity of the maps. Parameters are $\mu=4$ (equal for all maps), $\varepsilon_{1}=0.2$, and $\varepsilon_{2}=0.018$. The same initial and boundary conditions as in the caption of Fig. 1 .

If one further increases the coupling constant $\varepsilon_{2}$ far above the critical value for the emergence of WS, the system begins synchronizing the signals $\mathcal{M}^{i}(t) \quad(i=$ $1, \ldots, N)$ onto periodic states. More precisely, the synchronized state now becomes periodic, and recapitulates the bifurcation diagram of the single logistic map, from the chaotic state up to the period one state, which is realized at $\varepsilon_{2}=\frac{1}{8}$. The reason is that the coupling reduces the map parameter $\mu$ to $\tilde{\mu}=\mu\left(1-2 \varepsilon_{2}\right)$. Thus, the single map reenters the periodic cascade region for large values of $\varepsilon_{2}$, and the difference in $\varepsilon_{2}$ between period $Q$ and period $\frac{Q}{2}$ synchronized states shrinks by the same Feigenbaum factor $\delta \simeq 4.669$ [11].

Let us now discuss the robustness of the above scenario against small differences in the parameter values of the single maps. For this purpose, we consider in Eq. (1) the different $\mu_{k}^{i}$ parameters for each map. $\mu_{k}^{i}$ are randomly selected between maximal and minimal values $\left(\mu_{\min } \leq\right.$ $\left.\mu_{k}^{i} \leq \mu_{\max }\right)$ for all maps and for all lattices. Selecting $\mu_{\min }>\mu_{c}$ and $\mu_{\max } \leq 4$ means considering an ensemble of nonidentical chaotic logistic maps coupled by a global mean field interaction. The results are the same as in the case of identical maps: For all choices of $\mu_{\min }$ and $\mu_{\max }$, a critical value of $\varepsilon_{2}$ exists at which a sharp transition to WS occurs, with the same dynamical features as those reported in Figs. 1-3.

Finally, we show that WS behavior can be associated with spatial pattern formation. Spatial pattern formation is a feature of Eq. (1) as soon as $\varepsilon_{1} \neq 0$, implying a diffusive coupling among the maps of the same lattice, which may lead to the appearence of one-dimensional patterns of map activity. Such patterns are then coupled through a second collective interaction (that occurring at $\varepsilon_{2} \neq 0$ ), implying only mean field effects between lattices. Figure 4 shows that the appearence of two-dimensional patterns can be associated with WS phenomena.

In conclusion, we have shown that a phase synchronized state can emerge in the collective behavior of an ensemble of chaotic coupled map lattices, due to a mean field interaction. Such a new dynamical regime, and the transition to it, has been quantitatively studied in an ensemble of onedimensional lattices of logistic maps. The relevance of WS in parallel signal transmission and its relationship with pattern formation phenomena will be studied elsewhere.

The authors acknowledge A.S. Mikhailov and D.H. Zanette for fruitful discussions. This work was partly supported by Ministerio de Educacion y Ciencia, Spain (Grant No. PB95-0578), Universidad de Navarra, Spain (PIUNA), Integrated Action Italy-Spain HI97-30. F. S. de S. R. acknowledges financial support from the association "Amigos de la Universidad de Navarra." S. B. acknowledges financial support from EU Contract No. ERBFMBICT983466.

[1] L. M. Pecora and T.L. Carroll, Phys. Rev. Lett. 64, 821 (1990).

[2] S. Hayes et al., Phys. Rev. Lett. 73, 1781 (1994).

[3] K. M. Cuomo and A. V. Oppenheim, Phys. Rev. Lett. 71, 65 (1993); N. Gershenfeld and G. Grinstein, Phys. Rev. Lett. 74, 5024 (1995); Lj. Kocarev and U. Parlitz, Phys. Rev. Lett. 74, 5028 (1995); J. H. Peng et al., Phys. Rev. Lett. 76, 904 (1996); S. Boccaletti, A. Farini, and F. T. Arecchi, Phys. Rev. E 55, 4979 (1997).

[4] M. G. Rosenblum, A.S. Pikovsky, and J. Kurths, Phys. Rev. Lett. 76, 1804 (1996).

[5] M. G. Rosenblum, A.S. Pikovski, and J. Kurths, Phys. Rev. Lett. 78, 4193 (1997).

[6] E. Rosa, Jr., E. Ott, and M. H. Hess, Phys. Rev. Lett. 80, 1642 (1998).

[7] S. H. Strogatz, S.E. Mirollo, and P.C. Matthews, Phys. Rev. Lett. 68, 2730 (1992); J. F. Heagy, L. M. Pecora, and T. L. Carroll, Phys. Rev. Lett. 74, 4185 (1995).

[8] D. H. Zanette and A.S. Mikhailov, Phys. Lett. A 235, 135 (1997); D. H. Zanette, Phys. Rev. E 55, 5315 (1997); D. H. Zanette and A.S. Mikhailov, Phys. Rev. E (to be published).

[9] R. M. May, Nature (London) 261, 459 (1976).

[10] K. Kaneko, Phys. Rev. Lett. 65, 1391 (1990); Physica (Amsterdam) 55D, 368 (1992); G. Perez et al., Phys. Rev. A 45, 5469 (1992); S. Sinha et al., Phys. Rev. A 46, 3193 (1992); Phys. Rev. A 46, 6242 (1992); G. Perez, S. Sinha, and H. A. Cerdeira, Physica (Amsterdam) 63D, 341 (1993); S. Sinha, Phys. Rev. E 57, 4041 (1998).

[11] M. J. Feigenbaum, J. Stat. Phys. 19, 25 (1978); M. J. Feigenbaum, J. Stat. Phys. 21, 69 (1979). 